\section{Making Surface Replicas With Cellulose Acetate Sheets} Andrew W. Blackwood, Structure Probe, Inc.

The of use this material goes back to the days when fractography was done by TEM because SEM was not commercially available. There is, therefore, some very old literature on how to use the material, but since most of it is art, anyway, most current authors assume that the reader is generally familiar with the technique.

There are actually two purposes in using this material. Even for relatively small samples, it may be necessary to remove corrosion products in a nondestructive manner. In my opinion the best way to do this is to use acetone-softened cellulose acetate, which will remove the corrosion product but leave what is left of the metal. I know about various "nondestructive" chemical removal agents and I have done enough testing of these agents that I question whether they are truly nondestructive. We have one chance at a "real" failure.

The second purpose is to make a replica of the cleaned fracture surface for examination in the microscope. Whichever the purpose, the technique is basically the same.

For TEM replication, historic practice has generally been to dissolve the replicating material in a suitable solvent at a concentration around $2 \%$, put a drop on the area of interest, place a grid on the drop and go away for a while. Replicating materials with which I have some familiarity include collodion, Parlodion, Formvar, Butvar, Pioloform, poly(acrylic acid) and good old cellulose acetate. There is an appropriate solvent for each, and part of the "art" is selecting the proper combination. For example, water, used to dissolve poly(acrylic acid), is very suitable for some polymer surfaces but not suitable for most metals. When the solvent is completely evaporated, the grid can be picked up using tweezers, shadowed (or coated for SEM) and examined. This can work very well for TEM on the large, flat surfaces of those rare fatigue failures which wind up in textbooks. It also gives an unequivocal indication which part of the fracture surface generated which replica, but it has problems for "real" fractures in the SEM.

The basic problem is that the procedure is too good. The solution gets into every crack and crevice of the fracture surface, and the replica is "locked" to the surface so that it is impossible to remove without tearing. For practical SEM use, then, normal practice has been to use "tape".

Cellulose acetate is available from various suppliers (including SPI Supplies) as tape, a strip of material about $2.5 \mathrm{~cm}$ wide, from which the piece to be used is cut. Also available from some suppliers are sheet product. There tend to be different thickness preferences; all thicknesses work, but some folks like it thicker, and others like it thinner. In use, the area to be replicated is moistened with the solvent (acetone for cellulose acetate). The tape is also moistened on the side that will form the replica, and the solvent is allowed to soften the tape enough, but not too much. Yes, there is some "art" here! I find that forming a shallow "cup" in the tape helps me to hold the solvent in the area of interest without making a mess. The obvious idea of

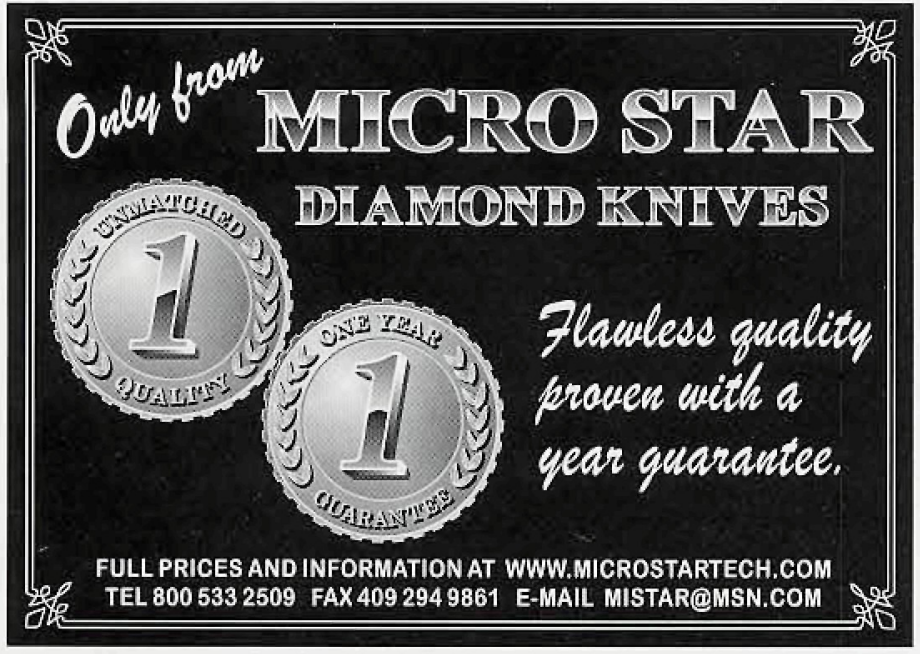

dipping the tape into the solvent is a recipe for a real mess, because the next step will fail.

When the tape has softened enough, but not too much, it is carefully pressed into the drop of solvent on the surface of interest. Thorough contact of wetted surfaces is essential. Our "official" tool for pressing is the eraser on a pencil but when the situation is serious, I use my right thumb. (I suppose that my left thumb would work, but l've never tried :-)). If the tape is too soft, the pressing tool will go through the tape, ruining the replica; if the tape is not soft enough, it will sit above the surface of interest and not capture its fine features. This is an art, not an "exact" science.

The next step is the most critical - go away for a while. Most "bad" replicas were destroyed by being "pulled" too quickly. It is essential that the solvent be completely evaporated, or the fine features will be left on the fracture surface. When completely dry, the replica is removed by pulling the tape upward. With the obvious exception that the tape may be attacked by the solvents in various "paint" products, the replica can be prepared like any other nonconductive SEM sample and examined at a remote location.

In actual practice, I do ten times as much replication to clean fracture surfaces as I do to examine them. The "first pull" replica from cleaning the fracture surface provides a "clean" sample of the corrosion product without any interference from the substrate. In some cases, this is actually a better procedure than attempting to analyze a corrosion product still on the substrate.

A Caveat: replication involves the use of volatile solvents, some of which are somewhat nasty. My first experience with TEM was making replicas using collodion dissolved in amyl acetate. Not knowing anything about amyl acetate at that time, I can only observe that I developed the habit of driving 90 miles an hour on the way home after breathing the stuff all day. Please be careful.

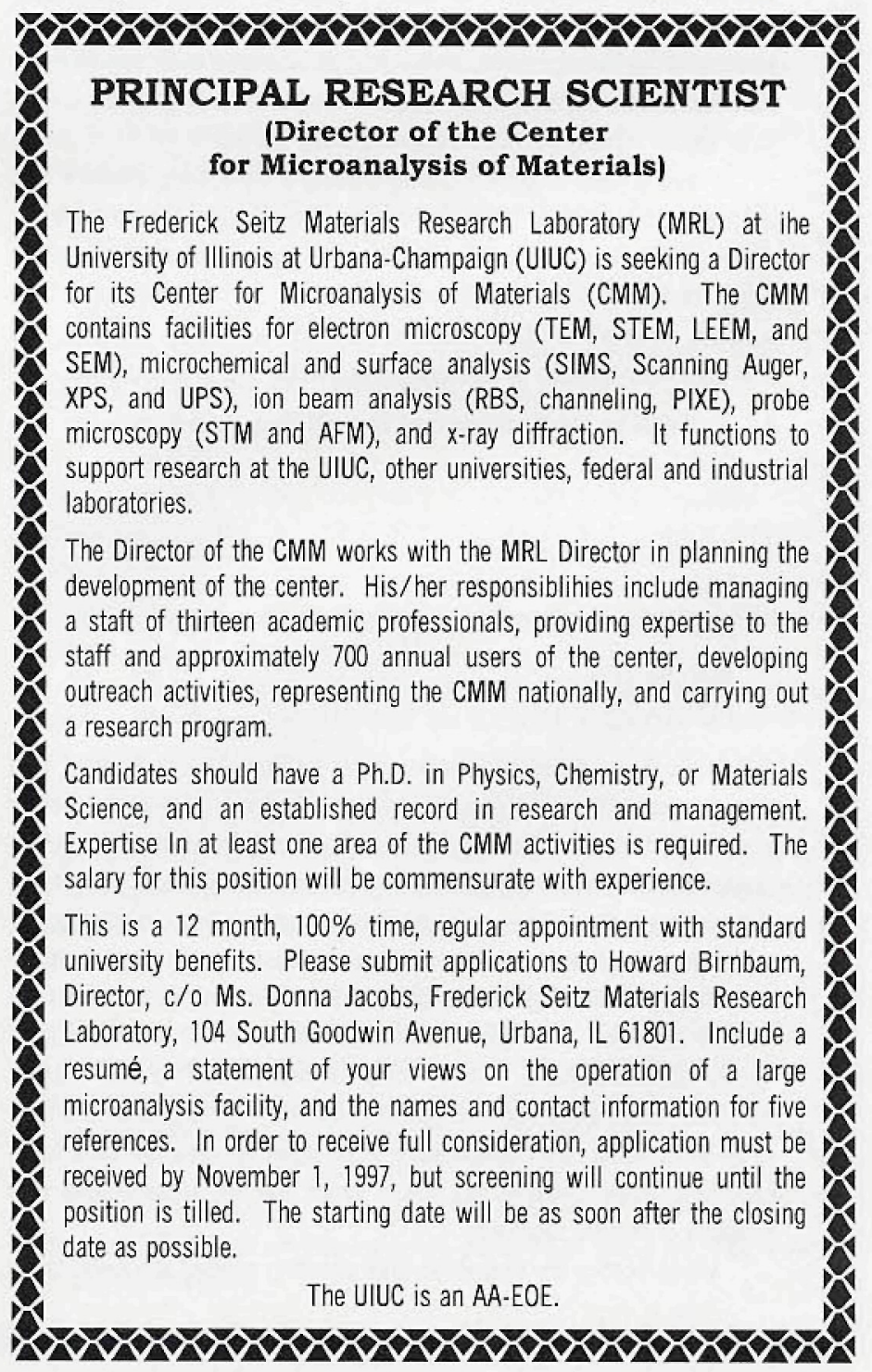

